\title{
TOEPLITZ-BEREZIN QUANTIZATION AND NON-COMMUTATIVE DIFFERENTIAL GEOMETRY
}

\author{
HARALD UPMEIER \\ Fachbereich Mathematik, Universität Marburg \\ Lahnberge, 35032 Marburg, Germany \\ E-mail: upmeier@mathematik.uni-marburg.de
}

In this survey article we describe how the recent work in quantization in multi-variable complex geometry (domains of holomorphy, symmetric domains, tube domains, etc.) leads to interesting results and problems in $\mathrm{C}^{*}$-algebras which can be viewed as examples of the "non-commutative geometry" in the sense of A. Connes. At the same time, one obtains new functional calculi (of pseudodifferential type) with possible applications to partial differential equations and group representations.

1. Geometry of phase spaces. The non-commutative geometry emerging in quantization theory arises as a deformation of the "classical" geometry of the underlying phase space, along a deformation parameter ("Planck's constant"). From the point of view of harmonic analysis, this parameter is related to representation theory of a "symmetry group" $G$ acting on phase space. This interplay between complex Kähler geometry, irreducible representations and $\mathrm{C}^{*}$-algebras is closest for the so-called symmetric domains.

We will first describe the geometry of symmetric phase spaces in algebraic terms. In the complex case (considered in this article) the appropriate objects are Jordan algebras and Jordan triples [FK, L1, U1].

Let $Z$ be a complex vector space of dimension $n$, endowed with a triple product

$$
Z \times \bar{Z} \times Z \ni(u, v, w) \mapsto\left\{u v^{*} w\right\} \in Z
$$

which is symmetric bilinear in $(u, w)$ and conjugate-linear in $v$. We say that $Z$ is a Jordan triple if the multiplication operators

$$
\left(u \square v^{*}\right) w:=\left\{u v^{*} w\right\}
$$

1991 Mathematics Subject Classification: 47B35, 32M15.

Research partially supported by NSF.

The paper is in final form and no version of it will be published elsewhere. 
acting on $Z$ satisfy the commutation relations

$$
\left[u \square v^{*}, x \square y^{*}\right]=\left\{u v^{*} x\right\} \square y^{*}-x \square\left\{y u^{*} v\right\}^{*}
$$

for all $u, v, x, y \in Z$. Define the Bergman operators

$$
B(u, v)=I-2 u \square v^{*}+2\left(u \square v^{*}\right)^{2}-\left\{u v^{*} u\right\} \square v^{*}
$$

so that, as a consequence of (1.1), we have

$$
B(u, v) z=z-2\left\{u v^{*} z\right\}+\left\{u\left\{v z^{*} v\right\}^{*} u\right\}
$$

for all $z \in Z$. A pair $(u, v) \in Z \times \bar{Z}$ is called quasi-invertible iff $B(u, v) \in G L(Z)$ is invertible. Put

$$
Z_{\text {reg }}:=\{z \in Z:(z, z) \text { quasi-invertible }\}=\{z \in Z: B(z, z) \in G L(Z)\} .
$$

This is an open dense subset of $Z$. We will always assume that the sesqui-linear "trace form"

$$
(u \mid v):=\operatorname{trace} u \square v^{*}
$$

which satisfies the "associativity" condition $\left(\left\{u v^{*} w\right\} \mid z\right)=\left(u \mid\left\{v w^{*} z\right\}\right)$ is non-degenerate. In the "irreducible" case of rank $r$, there exists a sesqui-polynomial $N_{r, r}(u, v)$ on $Z \times \bar{Z}$, called the Jordan triple determinant, such that $\operatorname{Det} B(u, v)=N_{r, r}(u, v)^{p}$ where $p$ is an integer called the genus.

A Jordan triple $Z$ is called (positive) hermitian if the scalar product (1.2) is positive definite. In this case the 0 -component of $Z_{\text {reg }}$ is the open unit ball

$$
B=\left\{z \in Z: I-z \square z^{*}>0\right\}
$$

for the so-called "spectral norm". Here $>0$ means positive definite with respect to (1.2). One can show that the group

$$
G=\operatorname{Aut}(B)^{0} \quad(\text { identity component) }
$$

of all biholomorphic automorphisms of $B$ is a semi-simple Lie group acting transitively on $B$, so that $B=G / K$ where $K=\{g \in G: g(0)=0\}$ is the stabilizer subgroup at $0 \in B$. Since $B$ is circular, $K$ consists of linear transformations preserving the Jordan triple product. In particular, $K$ preserves the inner product and is therefore compact. The pair $(G, K)$ is a hermitian symmetric pair making $B$ into a bounded symmetric domain.

The relationship between hermitian Jordan triples and symmetric domains is essentially one-to-one [L1, U1] and gives a Jordan theoretic refinement of the well-known Harish-Chandra realization of hermitian symmetric spaces of non-compact type, usually constructed in Lie-theoretic terms. For example, the curvature operator $R(u, v)$ which, for any symmetric space, gives rise to a "Lie triple product" on the tangent space $Z=T_{0}(B)$ can be expressed as

$$
R(u, v)=u \square v^{*}-v \square u^{*}
$$

for all $u, v \in Z$ [L1]. Thus the Jordan triple product can be regarded as the "positive part" of the curvature. Also, the inner product (1.2) is the $G$-invariant Kähler structure on $B$ evaluated at the "base point" $0 \in B$. The imaginary part of the Kähler metric is a $G$-invariant symplectic form, so that $B$ can be considered as a "classical phase space". 
EXAmPLE. Let $Z=\mathbb{C}^{r \times s}(r \leq s)$ be the space of all complex $(r \times s)$-matrices $z=\left(z_{i j}\right)$. Then the Jordan triple product on $Z$ is given by the generalized anti-commutator

$$
\left\{u v^{*} w\right\}:=\frac{1}{2}\left(u v^{*} w+w v^{*} u\right)
$$

which makes sense even for rectangular matrices. In operator terms, we have

$$
u \square v^{*}=\frac{1}{2}\left(L_{u v^{*}}+R_{v^{*} u}\right)
$$

(left and right matrix multiplicators) and the inner product

$$
\text { trace } u \square v^{*}=(r+s) \operatorname{trace}\left(u v^{*}\right)
$$

is positive definite. The Bergman operator is

$$
B(u, v)=\left(I-L_{u v^{*}}\right)\left(I-R_{v^{*} u}\right)
$$

where $I$ is the identity operator. Thus $B(u, v) z=\left(1-u v^{*}\right) z\left(1-v^{*} u\right)$ and $\operatorname{Det} B(u, v)=$ $\operatorname{Det}\left(1-u v^{*}\right)^{r+s}$. Hence the Jordan triple determinant is $N_{r, r}(u, v)=\operatorname{Det}\left(1-u v^{*}\right)$. The set $Z_{\text {reg }}=\left\{z \in Z: \operatorname{Det}\left(1-z z^{*}\right) \neq 0\right\}$ has the 0 -component $B=\left\{z \in Z: 1_{r}-z z^{*}>0\right\}$ which is just the unit ball of $Z$ in the operator norm. The group $G$ consists of all Moebius transformations

$$
\left(\begin{array}{ll}
a & b \\
c & d
\end{array}\right)(z):=(a z+b)(c z+d)^{-1}
$$

where $\left(\begin{array}{ll}a & b \\ c & d\end{array}\right)$ is a block matrix belonging to the pseudo-unitary group $U(r, s)$. The blockdiagonal matrices

$$
\left(\begin{array}{ll}
a & 0 \\
0 & d
\end{array}\right)(z)=a z d^{-1}
$$

with $a \in U(r), d \in U(s)$ induce linear transformations preserving the Jordan triple product. They constitute the subgroup $K$. Therefore $B=U(r, s) / U(r) \times U(s)$. The number $r$ is called the rank of $B$. For $r=1$, we obtain the Hilbert ball $B=\left\{z \in \mathbb{C}^{n}\right.$ : $z \cdot \bar{z}<1\}$ in $\mathbb{C}^{n}$. It is the only class of symmetric domains which have a smooth boundary.

An important class of Jordan triples comes from Jordan algebras. Let $X$ be a real vector space of dimension $n$, endowed with a commutative (but non-associative) product $x \circ y$. We say that $X$ is a Jordan algebra if the "multiplication operators"

$$
M_{x} y:=x \circ y
$$

acting on $X$ satisfy the commutation relations $\left[M_{x}, M_{x^{2}}\right]=0$ for all $x \in X$. Here $x^{2}:=$ $x \circ x$. Define the "quadratic representation" operator

$$
P_{x}:=2 M_{x}^{2}-M_{x^{2}} .
$$

Assume that $X$ has a unit element $e$. An element $x \in X$ is called invertible iff $P_{x} \in G L(X)$ is invertible. Put

$$
X_{\text {reg }}:=\{x \in X: x \text { invertible }\}=\left\{x \in X: P_{x} \in G L(X)\right\} .
$$

This is an open dense subset of $X$. We will always assume that the bilinear "trace form"

$$
(x \mid y):=\operatorname{trace} M_{x \circ y}
$$

which satisfies the "associativity" condition $(x \circ y \mid z)=(x \mid y \circ z)$ is non-degenerate. In the irreducible case, there exists a polynomial $N_{r}(x)$ on $X$ such that $N_{r}(e)=1$ and 
Det $P_{x}=N_{r}(x)^{n / r}$ where $n$ is the dimension and $r$ is the so-called rank. $N_{r}(x)$ is called the Jordan algebra determinant. It is related to, but different from, the triple determinant (which depends on two arguments). Another way to introduce $N_{r}$ is via "Cramer's rule"

$$
x^{-1}=\frac{\operatorname{grad} N_{r}(x)}{N_{r}(x)}
$$

where $x^{-1}$ is the Jordan algebra inverse and grad is the gradient with respect to (1.5).

A Jordan algebra $X$ is called (positive) euclidean if the trace form (1.5) is positive definite. In this case $X$ has a unit element $e$, and the $e$-component

$$
C=\left\{x \in X: M_{x}>0\right\}
$$

becomes a convex open cone which is symmetric in the sense that the group

$$
G L(C):=\{g \in G L(X): g C=C\}
$$

acts transitively on $C$, and $C$ is "self-dual" under the inner product (1.5) [FK]. One can show that $C$ can be endowed with the structure of Riemannian symmetric space such that

$$
S_{e} x:=x^{-1} \quad \text { (Jordan inverse) }
$$

is the "symmetry" around $e \in C$.

EXAMPLE. Let $\mathbb{K}$ be one of the real division algebras $\mathbb{R}, \mathbb{C}$ or $\mathbb{H}$ (quaternions), endowed with the usual involution. Then the space $X=H_{r}(\mathbb{K})=\left\{x \in \mathbb{K}^{r \times r}: x^{*}=x\right\}$ of all selfadjoint $(r \times r)$-matrices over $\mathbb{K}$ becomes a real Jordan algebra under the anti-commutator

$$
x \circ y=\frac{1}{2}(x y+y x) \text {. }
$$

Therefore $M_{x}=\frac{1}{2}\left(L_{x}+R_{x}\right)$ and the inner product trace $M_{x \circ y}=\frac{n}{r} \operatorname{Retrace}(x y)$ is positive definite. Note that the dimension $n=r+\operatorname{ar}(r-1) / 2$, where $a=1,2$, 4 is the real dimension of $\mathbb{K}$. The quadratic representation is $P_{x}=L_{x} R_{x}$ so that $P_{x} y=x y x$. Therefore Det $P_{x}=(\operatorname{Det} x)^{n / r}$ and the Jordan algebra determinant is $N_{r}(x)=\operatorname{Det}(x)$. The set $X_{\text {reg }}=\{x \in X:$ Det $x \neq 0\}$ has the $e$-component $C=\{x \in X: x>0\}$. The group $G L(C)$ consists of all linear transformations $g \cdot x=g x g^{*}$ with $g \in G L(r, \mathbb{K})$, and the stabilizer subgroup at $e \in C$ is given by $x \mapsto u x u^{*}$ where $u \in U(r, \mathbb{K})$ is unitary. Thus $C=G L(r, \mathbb{K}) / U(r, \mathbb{K})$ in this case.

For any real Jordan algebra $(X, \circ)$, the complexification $Z=X^{\mathbb{C}}$ has the Jordan triple product

$$
\left\{u v^{*} w\right\}=\left(u \circ v^{*}\right) \circ w+\left(w \circ v^{*}\right) \circ u-(u \circ w) \circ v^{*} .
$$

Here $u \circ w$ is the complexified Jordan algebra product on $Z$, and $v^{*}$ denotes the involution of $Z$ with respect to $X$. Assuming that $X$ has a unit element $e$, we have $X=\{x \in X$ : $\left.\left\{e x^{*} e\right\}=x\right\}$ and

$$
x \circ y=\left\{x e^{*} y\right\}
$$

for all $x, y \in X$. Thus the involution and the algebra product can be expressed in terms of the triple product (1.8). 
Example. For $n \geq 3, Z=\mathbb{C}^{n}$ has the Jordan triple product

$$
\left\{u v^{*} w\right\}=(u \cdot \bar{v}) w+(w \cdot \bar{v}) u-(u \cdot w) \bar{v}
$$

where $\bar{v}$ is the usual conjugation and $u \cdot w$ is the (bilinear) dot product. Note the formal analogy with (1.8). The Jordan triple automorphism group is $K=\mathbb{T} \cdot S O(n)$ where $\mathbb{T}$ acts by rotation. The corresponding symmetric domain is the "Lie ball"

$$
B=\left\{z \in \mathbb{C}^{n}: 1-z \cdot \bar{z}>0,1-2(z \cdot \bar{z})^{2}+|z \cdot z|^{2}>0\right\}
$$

which has rank 2 . The group $G=S O(n, 2)$ acts on $B$ via fractional "quadratic" transformations in a transitive way $[\mathrm{L} 1, \mathrm{U} 1]$ so that $B=S O(n, 2) / \mathbb{T} \cdot S O(n)$. Let $e=(1,0, \ldots, 0)$ be the "unit element". Applying (1.9) and (1.10), we obtain a real Jordan algebra $X=\mathbb{R} \times i \mathbb{R}^{n-1}$ with product

$$
\left(x_{1}, i x^{\prime}\right) \circ\left(y_{1}, i y^{\prime}\right)=\left(x_{1} y_{1}+x^{\prime} \cdot y^{\prime}, i\left(x_{1} y^{\prime}+y_{1} x^{\prime}\right)\right)
$$

for all $x_{1}, y_{1} \in \mathbb{R}$ and $x^{\prime}, y^{\prime} \in \mathbb{R}^{n-1}$. Note that $X$ is not the real form with respect to the conjugation $z \mapsto \bar{z}$, but with respect to the "involution" $z \mapsto\left\{e z^{*} e\right\}$. Computing the inverse

$$
\left(x_{1}, i x^{\prime}\right)^{-1}=\frac{\left(x_{1},-i x^{\prime}\right)}{x_{1}^{2}-x^{\prime} \cdot x^{\prime}}
$$

we obtain $N_{2}\left(x_{1}, i x^{\prime}\right)=x_{1}^{2}-x^{\prime} \cdot x^{\prime}$. Its complexification is $N_{2}(z)=z \cdot z$. The Jordan algebra $X$ is called a real spin factor. The condition $n \geq 3$ implies that $X$ is irreducible.

For domains of rank 1, namely the Hilbert ball in $\mathbb{C}^{n}$, the boundary is the (smooth) sphere $\partial B=S^{2 n-1}$. In all other cases the boundary is not smooth but has a stratification

$$
\partial B=\partial_{1} B \cup \ldots \cup \partial_{r} B .
$$

The $k$ th stratum $\partial_{k} B$ is a fibre bundle

$$
\partial_{k} B=\bigcup_{c \in S_{k}} c+B_{c}
$$

which is best described in terms of the underlying Jordan triple $Z$. An element $c \in Z$ is called a tripotent if $\left\{c c^{*} c\right\}=c$. Analogous to the idempotents in an algebra, one can write tripotents as an "orthogonal" sum

$$
c=c_{1}+\ldots+c_{k}
$$

of "minimal" tripotents and thus define the rank of $c$. The set $S_{k}$ of all tripotents of rank $k$ is a compact homogeneous manifold $S_{k}=K / L_{k}$ which we call the $k$ th skeleton of the boundary. For maximal rank $k=r$, we obtain the Shilov boundary $S=S_{r}=K / L_{r}$ which coincides with the extreme boundary of $B$. For $k \leq r$, the fibre over $c \in S_{k}$, called the boundary component determined by $c$, is a symmetric domain of rank $r-k$ (translated by $c$ ), which is given explicitly as the unit ball

$$
B_{c}=B \cap Z_{c}
$$

of the so-called Peirce 0 -space $Z_{c}:=\left\{z \in Z:\left\{c c^{*} z\right\}=0\right\}$. For maximal tripotents, we have $Z_{c}=\{0\}$ so that $\partial_{r} B=S_{r}$. 
ExAMPLE. For the matrix Jordan triple $Z=\mathbb{C}^{r \times s}(r \leq s)$ the tripotents are given by the equation $c=c c^{*} c$ which characterizes the partial isometries. Thus $S_{k}$ consists of all partial isometries of (matrix) rank $k \leq r$. In particular, for $r=s$ we have $S=S_{r}=U(r)$ (unitary group). Every partial isometry $c$ induces Hilbert space decompositions of $\mathbb{C}^{r}$ and $\mathbb{C}^{s}$, resp., such that

$$
c=\left(\begin{array}{cc}
1_{k} & 0 \\
0 & 0
\end{array}\right) .
$$

In terms of this decomposition the boundary component of $c$ is

Note that this set belongs to $\partial B$ if $k \geq 1$.

$$
c+B_{c}=\left\{\left(\begin{array}{cc}
1_{k} & 0 \\
0 & w
\end{array}\right):\|w\|<1\right\} .
$$

ExAmple. For the Lie ball $B \subset \mathbb{C}^{n}$ (cf. (1.11)), the non-zero tripotents fall into two classes: The maximal (rank 2) tripotents constitute the Lie sphere $S=S_{2}=\mathbb{T} \cdot \mathbb{S}^{n-1}$. For $n=4$, the Lie sphere is known as the "conformal universe". The minimal (rank 1) tripotents have the form

$$
c=\frac{x+i \xi}{2}
$$

with $x, \xi \in \mathbb{S}^{n-1}$ and $x \cdot \xi=0$. In other words, $S_{1}$ is isomorphic to the cosphere bundle $S_{1}=\mathbb{S}^{*}\left(\mathbb{S}^{n-1}\right)$ via the identification (1.13). The Peirce 0 -space of $(1.13)$ is $Z_{c}=\mathbb{C} \cdot \bar{c}$ with $\bar{c}=\frac{x-i \xi}{2}$, so that $B_{c}=\{\zeta \bar{c}:|\zeta|<1\}$ is a disk.

An important general fact is the Siegel domain realization of bounded symmetric domains. We will consider only the case of domains of "tube type". Let $Z$ be the hermitian Jordan triple arising by complexification of a euclidean Jordan algebra $X$ (cf. (1.8)). Let $B$ be the open unit ball of $Z$ given by (1.3). On the other hand, let $C$ be the positive cone of $X$ and consider the tube domain

$$
D=C+i X=\left\{z \in Z: z+z^{*} \in C\right\} .
$$

One can show that the Cayley transformation

$$
\gamma(z):=(e+z) \circ(e-z)^{-1}
$$

gives a biholomorphic mapping $\gamma: B \rightarrow D$ mapping $0 \in B$ to $e \in D$. The domain $D$ (called a "Siegel domain of the first kind") is the unbounded realization of $B$.

We will now point out possible generalizations concerning non-convex phase spaces. The set $X_{\text {reg }}$ of all invertible elements in a unital Jordan algebra $X$ has also other connected components. For example, if $X$ is euclidean the opposite cone

$$
-C=\left\{x \in X: M_{x}<0\right\}
$$

is the $(-e)$-component. It is also convex. For Jordan algebras of rank $>1$, there exist also non-convex components $V$ of $X$ which are "pseudo-symmetric" cones. This means that the group $G L(C)$, which can be described in terms of the Jordan triple determinant as

$$
G L(C)=\left\{g \in G L(X): N_{r}(g x)=N_{r}(g e) \cdot N_{r}(x)\right\}
$$

is still transitive on $V$, but the stabilizer of a point in $V$ is not compact so that $V=$ $G L(C) / H$ where $H$ is non-compact. Thus $V$ carries only a pseudo-Riemannian metric. 
These so-called "satellite" cones can be described in a uniform way using a "frame" $e_{1}, \ldots, e_{r}$ of mutually orthogonal minimal idempotents in $X$. For each $0 \leq k \leq r$ consider the idempotent

$$
e^{(k)}:=e_{1}+\ldots+e_{k}-e_{k+1}-\ldots-e_{r} .
$$

Then $e^{(0)}=-e, e^{(r)}=e$. The $k$ th satellite cone is $V_{k}:=G L(C) / H_{k}$ where $H_{k}=$ $\left\{g \in G L(C): g e^{(k)}=e^{(k)}\right\}$. The cones $V_{1}, \ldots, V_{r-1}$ are non-convex, and

$$
X_{\mathrm{reg}}=\bigcup_{0 \leq k \leq r} V_{k}
$$

EXAMPLE. Consider the 3-dimensional spin factor realized as a matrix Jordan algebra

$$
X=H_{2}(\mathbb{R})=\left\{x \in \mathbb{R}^{2 \times 2}: x^{t}=x\right\} .
$$

Then $X_{\text {reg }}=X \cap G L_{2}(\mathbb{R})$ has 3 connected components

$$
X_{\text {reg }}=V_{++} \cup V_{+-} \cup V_{--}
$$

according to the signs of the two eigenvalues of $x$. Here $C=V_{++},-C=V_{--}$but $V_{+-}$is a non-convex pseudo-symmetric cone. In suitable coordinates $V_{+-}$arises as the exterior of the double light cone. The non-convex cone $V_{+-}$has dimension 3 but its "slices" [G1] are 2-dimensional. Also, $V_{+-}$is not contractible but has non-trivial 1-homology.

A real Jordan algebra $X$ is called pseudo-euclidean (or semi-simple) if the trace form (1.5) is non-degenerate but may have negative eigenvalues. The euclidean Jordan algebras are a special case. It turns out that for any pseudo-euclidean algebra $X$ the regular set (1.4) decomposes into pseudo-symmetric cones

$$
X_{\text {reg }}=\bigcup_{\theta} V_{\theta}
$$

but in the non-euclidean case none of the cones $V_{\theta}$ are convex. An example is the full matrix algebra $X=\mathbb{R}^{r \times r}$ with anticommutator product $(1.7)$ and $N_{r}(x)=\operatorname{Det} x$. Thus

$$
X_{\mathrm{reg}}=G L_{r}(\mathbb{R})=G L_{r}^{+}(\mathbb{R}) \cup G L_{r}^{-}(\mathbb{R})
$$

decomposes into 2 non-convex cones according to the sign of the determinant. They carry an invariant pseudo-Riemannian metric.

For any satellite cone $V$ or, more generally, for any pseudo-symmetric cone $V$ in a pseudo-euclidean Jordan algebra $X$, the tube domain $D=V+i X \subset Z$ defined as in (1.14) is still homogeneous under holomorphic transformations (since $V$ is linearly homogeneous) but $D$ will always contain non-invertible elements so there is no analog of the symmetry (1.6). However, there is the following important

TheOrem. Let $V$ be a pseudo-symmetric cone. Then the tube domain $D$ is a dense open subset of a pseudo-symmetric (in fact, pseudo-Kähler) complex manifold $D \hookrightarrow$ $G_{\tau}:=G / G^{\tau}$ where the group $G$ can be defined explicitly in terms of the underlying Jordan algebra, and $G^{\tau}$ is the stabilizer of a point in $V$. Note that $G^{\tau}$ is non-compact.

We will call $G^{\tau}=: \widehat{D}$ the pseudo-symmetric hull of $D$. We have $D=\widehat{D}$ if and only if $V$ is convex. 
This theorem has essentially been proved case-by-case by Kaneyuki (for the satellite cones) [K1] and d'Atri-Gindikin (for pseudo-euclidean Jordan algebras) [AG]. A more uniform treatment using directly the underlying Jordan algebra has still to be worked out.

2. Harmonic analysis of state spaces. In quantization theory the basic idea is to replace the phase space (whose points are the "pure states" of the classical theory) by a complex Hilbert space whose elements, usually realized as "wave functions", represent the states of the quantum mechanical system. We will describe the Hilbert state spaces as representation spaces of the semi-simple Lie group $G$ underlying the phase space geometry ( $G$ serves as the "symmetry group" of the quantization procedure).

Let $B$ be an (irreducible) bounded symmetric domain of rank $r$ and genus $p$. For a real parameter $\lambda>p-1$ consider the Hilbert space

$$
H_{\lambda}^{2}(B):=\left\{h: B \rightarrow \mathbb{C} \text { holomorphic: } \int_{B}|h(z)|^{2} N_{r, r}(z, z)^{\lambda-p} d V(z)<\infty\right\}
$$

where $N_{r, r}$ is the Jordan triple determinant and $d V$ is Lebesgue measure. One can show that $H_{\lambda}^{2}(B)$ contains the polynomial algebra $P(Z)$ and in particular is non-trivial [FK, U5]. $H_{\lambda}^{2}(B)$ is called the $\lambda$ th Bergman space since it has a reproducing kernel

$$
E_{\lambda}(z, w)=N_{r, r}(z, w)^{-\lambda}
$$

specializing to the standard Bergman space for $\lambda=p$. This means that (putting a suitable normalization constant into the Lebesgue measure) we have

$$
h(z)=\int_{B} N_{r, r}(z, w)^{-\lambda} h(w) N_{r, r}(w, w)^{\lambda-p} d V(w)
$$

for all $h \in H_{\lambda}^{2}(B)$. The $\lambda$-Bergman space carries an irreducible (projective) representation

$$
\pi_{\lambda}\left(g^{-1}\right) h(z):=\operatorname{Det}\left(\partial_{z} g\right)^{\lambda / p} \cdot h(g(z))
$$

for all $g \in G, h \in H_{\lambda}^{2}(B)$ and $z \in B$. Here $\partial_{z} g$ is the complex derivative and we choose a branch of $\operatorname{Det}\left(\partial_{z} g\right)^{\lambda / p}$ on the (simply-connected) domain $B$. This representation belongs to the "holomorphic discrete series" (analytically continued) of the semi-simple Lie group $G$.

For any complex vector space $Z \approx \mathbb{C}^{n}$ with scalar product $(z \mid w)$ one can define the Segal-Bargmann (or Fock) space

$$
H_{\hbar}^{2}(Z):=\left\{h: Z \rightarrow \mathbb{C} \text { holomorphic }: \int_{Z}|h(z)|^{2} e^{-(z \mid z) / \hbar} d V(z)<\infty\right\} .
$$

Here $\hbar>0$ is "Planck's constant" which can also be interpreted as the parameter of the "complex wave representation" of the $(2 n+1)$-dimensional Heisenberg group. The corresponding Kähler geometry is flat, so it cannot be directly related to the "curved" geometry of symmetric spaces but it can be obtained by deformation of the bounded realization $B$ by considering $R \cdot B(R>0)$ for $R \rightarrow \infty$. The reproducing kernel of $H_{\hbar}^{2}(Z)$ is given by plane waves

$$
E_{\hbar}(z, w)=e^{(z \mid w) / \hbar}
$$


The algebra $P(Z)$ of all polynomials on $Z$ is a dense subspace of $H_{\hbar}^{2}(Z)$ and, for $p, q \in$ $P(Z)$, the inner product

$$
(p \mid q)_{Z}:=\pi^{-n} \int_{Z} \overline{p(z)} q(z) e^{-(z \mid z)} d V(z)
$$

(taking $\hbar=1$ ) coincides with the "Fischer" inner product

$$
(p \mid q)_{Z}=\left(\partial_{p} q\right)(0)
$$

Here $\partial_{p}$ is the constant coefficient differential operator associated with $p$ via the scalar product.

A crucial fact concerning the $\lambda$-Bergman spaces is the Fourier decomposition under the action of $K[\mathrm{~S} 1, \mathrm{U} 2]$. Let $\alpha=\left(\alpha_{1}, \ldots, \alpha_{r}\right)$ be a "signature" of integers $\alpha_{1} \geq \ldots \geq \alpha_{r} \geq 0$. It is known [U2] that the "conical polynomial"

$$
N_{\alpha}(z)=N_{1}(z)^{\alpha_{1}-\alpha_{2}} N_{2}(z)^{\alpha_{2}-\alpha_{3}} \ldots N_{r}(z)^{\alpha_{r}}
$$

generates an irreducible $K$-module $P^{\alpha}(Z) \subset P(Z)$. Here $N_{1}, \ldots, N_{r}$ are the "minors" on $Z$ defined in terms of the Peirce decomposition [U2]. For example, $N_{r}$ is the Jordan algebra determinant for tube type domains. The decomposition

$$
P(Z)=\sum_{\alpha_{1} \geq \alpha_{2} \geq \ldots \geq \alpha_{r} \geq 0}^{\oplus} P^{\alpha}(Z)
$$

is multiplicity-free and orthogonal with respect to the canonical inner product (2.3) on $P(Z)$.

THEOREM. We have an orthogonal decomposition

$$
H_{\lambda}^{2}(B)=\sum_{\alpha_{1} \geq \ldots \geq \alpha_{r} \geq 0} P^{\alpha}(Z) \quad \text { (Hilbert sum) }
$$

such that for all $p, q \in P^{\alpha}(Z)$,

$$
\frac{(p \mid q)_{Z}}{(p \mid q)_{\lambda}}=((\lambda))_{\alpha}
$$

where

$$
((\lambda))_{\alpha}=\prod_{j=1}^{r}\left(\lambda-\frac{a}{2}(j-1)\right)_{\alpha_{j}}=\prod_{j=1}^{r} \prod_{i=0}^{\alpha_{j}-1}\left(\lambda-\frac{a}{2}(j-1)+i\right)
$$

is the "multi-Pochhammer" symbol. Here a is a fixed parameter.

For a detailed proof, cf. [FK, U5]. A far-reaching generalization is the well-known "Blattner's conjecture", proved in [S2], concerning the $K$-decomposition of any discrete series representation of $G$.

EXAMPLE. For the tube type domains of rank 2, the so-called Lie balls (1.11), the decomposition (2.4) takes the form

$$
H_{\lambda}^{2}(B)=\sum_{\alpha_{1} \geq \alpha_{2} \geq 0} P^{\alpha}\left(\mathbb{C}^{n}\right)
$$


and the conical polynomial $N_{\alpha}(z)$, which characterizes the $K$-module $P^{\alpha}\left(\mathbb{C}^{n}\right)$ as its highest weight vector, is given explicitly as

$$
N_{\alpha}(z)=\left(z_{1}-i z_{2}\right)^{\alpha_{1}-\alpha_{2}}(z \cdot z)^{\alpha_{2}} .
$$

These polynomials are closely related to the Gegenbauer polynomials.

The decomposition (2.4) concerns the bounded realization $B$ for which the stabilizer subgroup $K$ is linear. If we pass to the unbounded realization $D$ (here we consider only tube domains) there is also a $\lambda$-Bergman space $H_{\lambda}^{2}(D)$ of holomorphic functions on $D$ which has the reproducing kernel

$$
E_{\lambda}(z, w)=N_{r}\left(\frac{z+w^{*}}{2}\right)^{-\lambda}
$$

for all $z, w \in D$, where $N_{r}$ is the Jordan algebra determinant. The two Bergman type spaces are unitarily equivalent via the Cayley transform (1.15). However, in the unbounded case, it is more suitable to consider the Laplace transform

$$
\left(\mathcal{L}_{\lambda} \phi\right)(z)=c_{\lambda} \int_{C} e^{-(z \mid \xi)} \phi(\xi) N_{r}(\xi)^{(\lambda-n / r) / 2} d \xi
$$

over the convex cone $C$. Here $N_{r}(\xi)^{-n / r} d \xi$ is the invariant measure on $C$ and $(z \mid \xi)$ is the trace form (1.5). The constant $c_{\lambda}$ is related to Gindikin's multi-variable $\Gamma$-function $[\mathrm{FK}$, $\mathrm{UU}]$.

Theorem. The Laplace transform induces a unitary equivalence $\mathcal{L}_{\lambda}: L^{2}(C) \rightarrow$ $H_{\lambda}^{2}(D)$.

Up to now, the state spaces were constructed as certain $G$-invariant Hilbert spaces of holomorphic functions. This corresponds geometrically to the fact that the underlying phase space $(B$, resp. $D)$ is convex and therefore a domain of holomorphy. If one replaces $C$ by a non-convex satellite cone $V$ (or, more generally, by any pseudo-symmetric cone), the tube domain $D=V+i X$ or its pseudo-symmetric hull $\widehat{D}$ are no longer pseudo-convex in the sense of complex analysis and therefore do not carry interesting holomorphic function spaces. Recently it has been shown by Gindikin [G1] that instead one has to look at (Dolbeault or Čech) cohomology classes.

Let $V \subset X_{\text {reg }}$ be a pseudo-symmetric cone of dimension $n$. Let $p$ be the dimension of the largest convex subspace ("slice") and put $q:=n-p$. Consider the open covering

$$
\left\{V_{\sigma}^{\#}+i X: V_{\sigma} \text { slice of } V\right\}
$$

of $V+i X$, where $V_{\sigma}^{\#}$ is the so-called dual cone $\left(V=V^{\#}\right.$ is self-dual). Then we define Čech $q$-cocycles

$$
h^{\sigma_{0}, \ldots, \sigma_{q}} \in O\left(\bigcap_{j=0}^{q}\left(V_{\sigma}^{\#}+i X\right)\right) \quad \text { (holomorphic functions) }
$$

with vanishing coboundary

$$
(\partial h)^{\sigma_{0}, \ldots, \sigma_{q+1}}:=\left.\sum_{i=0}^{q+1}(-1)^{i} h^{\sigma_{0}, \ldots, \hat{\sigma}_{i}, \ldots, \sigma_{q+1}}\right|_{\bigcap_{j=0}^{q+1}\left(V_{\sigma_{j}}^{\#}+i X\right)}=0 .
$$


Let $O_{q}(D)$ be the corresponding $q$-cohomology group. Since (2.6) is a Stein covering, the cohomology classes can also be expressed more explicitly using the $\bar{\partial}$-operator (Dolbeault cohomology). With this notation we have [G1]

TheOREM. There exists a Hardy-type completion $H_{q}^{2}(D)$ of $q$-cocycles which can actually be defined on the pseudo-symmetric hull $\widehat{D}$ of $D$ such that the boundary evaluation map together with the Laplace transform yields a unitary equivalence

$$
\mathcal{L}:\left.L^{2}(V) \gtrsim H_{q}^{2}(D)\right|_{i X} \quad \text { (restriction) }
$$

3. Toeplitz operator algebras and Berezin quantization. The reproducing kernel property of $E_{\lambda}(z, w)=N_{r, r}(z, w)^{-\lambda}$ (cf. (2.1)) means that the functions on $B$ defined by

$$
k_{w}(z):=E_{\lambda}(z, w) / E_{\lambda}(w, w)^{1 / 2}=N_{r, r}(z, w)^{-\lambda} N_{r, r}(w, w)^{\lambda / 2}
$$

for fixed $w \in B$ form an "overcomplete basis" of $H_{\lambda}^{2}(B)$ in the sense that

$$
h=\int_{B}\left(k_{w} \mid h\right)_{\lambda} k_{w} N_{r, r}(w, w)^{-p} d V(w)
$$

for each $h \in H_{\lambda}^{2}(B)$. In operator terms, one obtains a resolution of the identity operator

$$
I=\int_{B} k_{w} \otimes k_{w}^{*} N_{r, r}(w, w)^{-p} d V(w)
$$

where $\left(k_{w} \otimes k_{w}^{*}\right) h:=k_{w}\left(k_{w} \mid h\right)_{\lambda}$ is the usual rank one operator, linear in $h$. This idea leads to the concept of Berezin symbol of a (bounded, linear) operator $T$ on $H_{\lambda}^{2}(B)$, defined as the "diagonal"

$$
\sigma_{\lambda}(T)(w):=\left(k_{w} \mid T k_{w}\right)_{\lambda}=\operatorname{trace} T\left(k_{w} \otimes k_{w}^{*}\right)
$$

for all $w \in B$. As an explicit integral formula, we have

$$
\begin{aligned}
\sigma_{\lambda}(T)(w) & =\int_{B} \overline{k_{w}(z)}\left(T k_{w}\right)(z) N_{r, r}(z, z)^{\lambda-p} d V(z) \\
& =\int_{B} N_{r, r}(w, z)^{-\lambda}\left(T N_{r, r}(-, w)^{-\lambda}\right)(z) N_{r, r}(w, w)^{\lambda} N_{r, r}(z, z)^{\lambda-p} d V(z) .
\end{aligned}
$$

We will also call $\sigma_{\lambda}(T)$ the passive symbol of $T$. The transformation property

$$
\pi_{\lambda}\left(g^{-1}\right) k_{w}=k_{g w}
$$

of the normalized kernel vectors shows that $\sigma_{\lambda}$ intertwines the "classical" action of $\mathrm{G}$ on phase space and the "quantum" action (2.2) on state space: For all $g \in G$ and operators $T$ we have

$$
\sigma_{\lambda}(T) \circ g=\sigma_{\lambda}\left(\operatorname{Ad} \pi_{\lambda}\left(g^{-1}\right) T\right),
$$

where Ad is the adjoint representation. The Kähler geometry of $B$ defines a $G$-invariant "Liouville" measure

$$
d \mu(z)=N_{r, r}(z, z)^{-p} d V(z)
$$

on $B$, and thus gives rise to the Lebesgue space $L^{2}(B)$ with its unitary (unweighted) translation action of $G$. On the other hand, consider the Hilbert-Schmidt inner product $(S \mid T):=\operatorname{trace} S^{*} T$ on the space $\mathcal{L}^{2}\left(H_{\lambda}^{2}(B)\right)$ of all Hilbert-Schmidt operators. The 
Berezin quantization map is constructed as the adjoint of the symbol map $\sigma_{\lambda}$, with respect to the inner products described above. More precisely, let $f \in L^{\infty}(B)$ and put $T_{\lambda}(f) h:=E_{\lambda}(f h)$ for all $h \in H_{\lambda}^{2}(B)$, where $E_{\lambda}$ is the orthogonal projection defined by the reproducing kernel (2.1). More explicitly,

$$
\begin{aligned}
T_{\lambda}(f) h(z) & =\int_{B} E_{\lambda}(z, w) f(w) h(w) N_{r, r}(w, w)^{\lambda-p} d V(w) \\
& =\int_{B} N_{r, r}(z, w)^{-\lambda} f(w) h(w) N_{r, r}(w, w)^{\lambda-p} d V(w) \\
& =\int_{B} N_{r, r}(z, w)^{-\lambda} N_{r, r}(w, w)^{\lambda} f(w) h(w) d \mu(w)
\end{aligned}
$$

for all $z \in B$. The bounded linear operator $T_{\lambda}(f)$ on $H_{\lambda}^{2}(B)$ is called the Toeplitz operator with active Berezin symbol $f$, and the assignment $f \mapsto T_{\lambda}(f)$ is called Berezin quantization. It is easy to show [UU] that

$$
\operatorname{trace} T^{*} T_{\lambda}(f)=\int_{B} \overline{\sigma_{\lambda}(T)} f d \mu
$$

for suitable operators $T$ and functions $f$, which explains the duality mentioned above. As a consequence, or by direct calculation, it follows that

$$
T_{\lambda}(f \circ g)=\operatorname{Ad} \pi_{\lambda}\left(g^{-1}\right) T_{\lambda}(f)
$$

whenever $g \in G$. Here $\pi_{\lambda}$ is the projective representation (2.2).

In the corresponding flat situation $Z=\mathbb{C}^{n}$, with Planck's constant $\hbar>0$ and the associated Segal-Bargmann space $H_{\hbar}^{2}(Z)$, the Berezin type quantization leads to the socalled Wick calculus. Thus (putting a normalization constant into the Lebesgue measure)

$$
\begin{aligned}
T_{\hbar}(f) h(z) & =E_{\hbar}(f h)(z)=\int_{Z} E_{\hbar}(z, w) f(w) h(w) e^{-(w \mid w) / \hbar} d V(w) \\
& =\int_{Z} e^{(z \mid w) / \hbar-(w \mid w) / \hbar} f(w) h(w) d V(w)
\end{aligned}
$$

defines the operator $T_{\hbar}(f)$ on $H_{\hbar}^{2}(Z)$ with "active" Wick symbol $f \in L^{\infty}(Z)$, whereas

$$
\begin{aligned}
\sigma_{\hbar}(T)(w) & =\left(k_{w} \mid T k_{w}\right)_{\hbar}=\int_{Z} \overline{k_{w}(z)}\left(T k_{w}\right)(z) e^{-(z \mid z) / \hbar} d V(z) \\
& =\int_{Z} e^{(w \mid z) / \hbar} T\left(e^{(. \mid w) / \hbar}\right)(z) e^{-(w \mid w) / \hbar-(z \mid z) / \hbar} d V(z)
\end{aligned}
$$

is the "passive" Wick symbol of an operator $T$ on $H_{\hbar}^{2}(Z)$. One can show that the Wick calculus arises in a formal way as a limit of the Berezin calculus on the unit ball $B$ of $Z$, by letting the radius of $B$ tend to infinity.

The theory of Berezin quantization and its connections to operator theory, complex analysis and harmonic analysis (discrete series representations of $G$ ) form an active area of current research. We will concentrate on two results that elucidate the operator-theoretic aspects of Berezin quantization.

Given two Berezin type operators with active symbols $f$ and $g$, resp., one may form the operator $T_{\lambda}(f) T_{\lambda}(g)$ and ask for its (active or passive) symbol which will depend on 
$f, g$ and $\lambda$. In quantization theory such bilinear compositions are called $*$-products (or Moyal products) and denoted by $f * g$. Formally,

$$
T_{\lambda}(f) T_{\lambda}(g)=T_{\lambda}(f * g)
$$

but $f * g$ may only exist as an asymptotic series in $1 / \lambda$, and the coefficients of this expansion are not explicitly known (except for the Weyl or Wick calculus in the flat case). However, the first two terms of the expansion (which are physically most relevant) can be recovered in the strongest possible way (operator norm convergence) as $\lambda \rightarrow \infty$ :

Theorem [BLU]. For suitable smooth symbols $f, g$ on $B$, we have, as $\lambda \rightarrow \infty$,

$$
\left\|T_{\lambda}(f) T_{\lambda}(g)-T_{\lambda}(f g)\right\| \rightarrow 0, \quad\left\|\lambda\left[T_{\lambda}(f), T_{\lambda}(g)\right]-i T_{\lambda}(\{f, g\})\right\| \rightarrow 0,
$$

where $[S, T]$ is the operator commutator and $\{f, g\}$ is the invariant Poisson bracket.

Thus the usual product $f g$ and the Poisson bracket $\{f, g\}$ give the 0th and 1st order term of the expansion in $1 / \lambda$. It would be very interesting to find higher differential analogues.

The next result concerns $\mathrm{C}^{*}$-algebraic properties of the Berezin quantization procedure. Consider the Toeplitz $\mathrm{C}^{*}$-algebra

$$
\mathcal{T}_{\lambda}(B)=C^{*}\left(T_{\lambda}(f): f \in \mathcal{C}(\bar{B})\right)
$$

generated by all $\lambda$-Toeplitz operators with continuous symbol on the closure $\bar{B}$. A similar (but different) $\mathrm{C}^{*}$-algebra can be defined in the unbounded case by the additional requirement that the symbols vanish at infinity. The $\mathrm{C}^{*}$-algebra $\mathcal{T}_{\lambda}(B)$ represents the "non-commutative" geometry of the underlying phase space $B$, including the boundary. As described in Section 1, the boundary has a natural stratification given by the boundary components. The next result (originally proved for the case of Hardy space Toeplitz operators) shows that the stratification of the boundary induces a natural filtration of the associated Toeplitz $\mathrm{C}^{*}$-algebra.

TheOREM [U3, U4, U5]. Let B be an (irreducible) bounded symmetric domain of rank $r$. Then the Toeplitz $\mathrm{C}^{*}$-algebra $\mathcal{T}_{\lambda}(B)$ has a natural filtration

$$
\mathcal{K}=\mathcal{I}_{1} \triangleleft \mathcal{I}_{2} \triangleleft \ldots \triangleleft \mathcal{I}_{r} \triangleleft \mathcal{T}_{\lambda}(B)
$$

into $\mathrm{C}^{*}$-ideals, with associated graduation

$$
\mathcal{I}_{k+1} / \mathcal{I}_{k} \approx \mathcal{C}\left(S_{k}\right) \quad \text { (stably isomorphic) }
$$

for $0 \leq k \leq r$ being essentially commutative.

Here we put $\mathcal{I}_{r+1}:=\mathcal{T}_{\lambda}(B)$ and $S_{0}:=\{0\} . \mathcal{K}$ denotes the compact operators.

The essential geometric meaning of this result is that the irreducible representations of $\mathcal{T}_{\lambda}(B)$ (modulo unitary equivalence) are in one-to-one correspondence with the tripotents of $Z$ (including 0 ). In fact, for $c \in S_{k}$, the corresponding representation $\tau_{c}$ can be explicitly realized on the Bergman space $H_{\mu}^{2}\left(B_{c}\right)$ of the boundary component (1.12) given by $c$ :

$$
\tau_{c}: \mathcal{T}_{\lambda}(B) \rightarrow \mathcal{L}\left(H_{\mu}^{2}\left(B_{c}\right)\right)
$$

is uniquely characterized by the property $\tau_{c}\left(T_{\lambda}(f)\right)=T_{\mu}\left(f_{c}\right)$ for all $f \in \mathcal{C}(\bar{B})$, where $f_{c}(w):=f(c+w)$ denotes the restriction of $f$ to the fibre $c+B_{c}$ (here continuity up to 
the boundary is essential) and $\mu$ is a parameter depending on $\lambda$ which is adapted to the lower-dimensional geometry of $B_{c}$. For maximal tripotents, we obtain the characters

$$
\tau_{c}\left(T_{\lambda}(f)\right)=f(c) \quad\left(c \in S_{r}\right)
$$

which form the classical Toeplitz-type symbol familiar from the disk or the Hilbert ball.

The filtration (3.4) of $\mathcal{T}_{\lambda}(B)$ gives rise to index mappings

$$
\operatorname{Ind}_{k}: K^{1}\left(S_{k}\right) \rightarrow K^{0}\left(S_{k-1}\right)
$$

defined in terms of $K$-theory and related to the Atiyah-Singer family index. In [U5], these analytic indices are expressed topologically using the geometry of the boundary. Only the minimal tripotents $(k=1)$ give rise to a $\mathbb{Z}$-valued index

$$
\operatorname{Ind}_{1}: K^{1}\left(S_{1}\right) \rightarrow \mathbb{Z}
$$

since $S_{0}=\{0\}$. The space $S_{1}$ is a Stiefel type manifold but its $K$-theory has not been calculated in a uniform way.

ExAMPLE. For the Lie ball (1.11), the filtration (3.4) and associated index mappings can be expressed in terms of pseudo-differential operators. We have a filtration $\mathcal{K} \triangleleft \mathcal{I}_{2} \triangleleft$ $\mathcal{T}_{\lambda}(B)$ such that $\mathcal{T}_{\lambda}(B) / \mathcal{I}_{2} \cong \mathcal{C}\left(\mathbb{T} \cdot \mathbb{S}^{n-1}\right)$ and

$$
\mathcal{I}_{2} / \mathcal{K} \approx \mathcal{C}\left(\mathbb{S}^{*}\left(\mathbb{S}^{n-1}\right)\right) \quad(\text { stable isomorphism }) .
$$

One can show [U5] that $\mathcal{I}_{2}$ can be realized as the $\mathrm{C}^{*}$-algebra of Calderón-Zygmund operators (i.e., pseudo-differential operators of order 0 ) on $\mathbb{S}^{n-1}$. Via this identification, the mapping $\operatorname{Ind}_{1}: K^{1}\left(\mathbb{S}^{*}\left(\mathbb{S}^{n-1}\right)\right) \rightarrow \mathbb{Z}$ is the Atiyah-Singer index.

4. Berezin transform and invariant differential operators. Combining the Berezin symbol map $\sigma_{\lambda}$ and its adjoint, the Berezin quantization map, we obtain the Berezin transform

$$
\sigma_{\lambda}\left(T_{\lambda}(f)\right)(w)=\left(k_{w} \mid f k_{w}\right)_{\lambda}
$$

of a (bounded measurable) function $f$ on $B$. By (3.1) and (3.2), we have

$$
\sigma_{\lambda}\left(T_{\lambda} f\right) \circ g=\sigma_{\lambda}\left(T_{\lambda}(f \circ g)\right)
$$

for all $g \in G$, i.e., the Berezin transform commutes with the (unweighted) translation action of $G$. Restricting to smooth functions, it should therefore be expressible in terms of (scalar) $G$-invariant differential operators.

In the case of Wick quantization (cf. (3.3)), corresponding to the flat phase space $Z=\mathbb{C}^{n}$ with Kähler metric $(z \mid w)=z \cdot \bar{w}$, this relationship is well-known [G2]: For Planck's constant $\hbar>0$ we have

$$
\sigma_{\hbar}\left(T_{\hbar}(f)\right)=e^{\hbar \Delta} f
$$

where

$$
\Delta=\sum_{i} \frac{\partial^{2}}{\partial z_{i} \partial \bar{z}_{i}}
$$

is the non-positive Laplace-Beltrami operator of $Z$. Thus the Berezin transform is essentially the heat semigroup on $Z$. This relationship has important consequences, in particular for the connection between the Wick calculus and the Weyl calculus (usually defined 
on the real wave space $L^{2}\left(\mathbb{R}^{n}\right)$ ), and for characterizing growth properties of operators in terms of their symbols.

For any symmetric domain (in its bounded or unbounded) realization there exists a unique $G$-invariant Laplace-Beltrami operator $\Delta$ which is defined in terms of the $G$ invariant Riemannian metric (the real part of the Kähler metric). For example, the unit disk has the Laplace-Beltrami operator

$$
\Delta=\left(1-|z|^{2}\right)^{2} \frac{\partial^{2}}{\partial z \partial \bar{z}}
$$

Since in general $\Delta$ is a differential operator of order 2 involving $z$-derivatives and $\bar{z}$ derivatives in a balanced way, we write $\Delta=\Delta_{1,1}$. However, for domains of rank $>1$, not every $G$-invariant differential operator is a polynomial in $\Delta$. More precisely, for an irreducible symmetric domain $G / K$ of rank $r$, the algebra $D_{G}(G / K)$ of all scalar $G$-invariant differential operators on $G / K$ is commutative and has $r$ algebraically independent generators $\Delta_{1,1}, \Delta_{2,2}, \ldots, \Delta_{r, r}$ of order $2,4, \ldots, 2 r$. Thus

$$
D_{G}(G / K)=\mathbb{C}\left[\Delta_{1,1}, \Delta_{2,2}, \ldots, \Delta_{r, r}\right] .
$$

These operators are called the "higher Laplacians" of $G / K$, since $\Delta_{1,1}$ is the usual Laplace-Beltrami operator. It is unique up to a constant. The other generators are not canonical but one can choose appropriate generators by prescribing their "radial parts" with respect to the Iwasawa decomposition $G=K A N$.

Since the operators $\Delta_{1,1}, \ldots, \Delta_{r, r}$ commute with each other, one may use the functional calculus to form functions $f\left(\Delta_{1,1}, \ldots, \Delta_{r, r}\right)$ of these operators. In view of $(4.2)$, it is natural to expect that for symmetric domains of rank $r$ the Berezin transform (4.1) will be an operator function of this type for a suitable multi-variable $f\left(t_{1}, \ldots, t_{r}\right)$. This idea was carried out by Berezin [B1] for the classical spaces (without proof), and in general in [UU]. The proof in [UU] does not use the classification of symmetric domains and establishes the result in the strongest, spectral-theoretic, sense:

TheOREM [UU]. Let $B=G / K$ be an irreducible symmetric domain of rank $r$, and choose a basis $\Delta_{k, k}(1 \leq k \leq r)$ of $G$-invariant differential operators on $B$ such that the (orispherical) radial part of $\Delta_{k, k}$ corresponds to the (even symmetric) polynomial $t_{1}^{2 k}+\ldots+t_{r}^{2 k}$. Then the Berezin transform is given by

$$
\sigma_{\lambda}\left(T_{\lambda}(f)\right)=\Gamma_{\lambda}\left(\Delta_{1,1}, \ldots, \Delta_{r, r}\right) f
$$

where

$$
\Gamma_{\lambda}\left(t_{1}, \ldots, t_{r}\right)=\prod_{j=1}^{r} \frac{\Gamma\left(\lambda-\frac{p-1}{2}+i t_{j}\right) \Gamma\left(\lambda-\frac{p-1}{2}-i t_{j}\right)}{\Gamma\left(\lambda-\frac{p-1}{2}+\rho_{j}\right) \Gamma\left(\lambda-\frac{p-1}{2}-\rho_{j}\right)} .
$$

Here $\Gamma$ is Euler's $\Gamma$-function, $p$ is the "genus" of $B$ and $\left(\rho_{1}, \ldots, \rho_{r}\right)$ corresponds to the half-sum of positive roots of $(G, K)$.

The main point of this theorem is that a multi-variable $\Gamma$-type function replaces the exponential in (4.2) when passing to the case of bounded symmetric domains. The occurrence of multivariable special functions (of hypergeometric type) is an attractive feature of quantization theory of symmetric spaces. For example, the on-going investigation of Berezin transforms in the vector-valued setting (discrete series representations of $G$ ) or 
for the Weyl-type quantization studied in [U6,U7] leads to novel multivariable special functions closely related to the harmonic analysis of the underlying phase spaces.

\section{References}

[AG] J. d'Atri and S. Gindikin, Siegel domain realization of pseudo-Hermitian symmetric manifolds, Geom. Dedicata 46 (1993), 91-125.

[B1] F. A. Berezin, A connection between the co- and contravariant symbols of operators on classical complex symmetric spaces, Soviet Math. Dokl. 19 (1978), 786-789.

[BLU] D. Borthwick, A. Lesniewski and H. Upmeier, Non-perturbative deformation quantization of Cartan domains, J. Funct. Anal 113 (1993), 153-176.

[FK] J. Faraut and A. Korányi, Analysis on Symmetric Cones, Oxford Univ. Press, 1994.

[G1] S. Gindikin, Fourier transform and Hardy spaces of $\bar{\partial}$-cohomology in tube domains, C. R. Acad. Sci. Paris Sér. I Math. 315 (1992), 1139-1143.

[G2] V. Guillemin, Toeplitz operators in n-dimensions, Integral Equations Operator Theory 7 (1984), 145-205.

[K1] S. Kaneyuki, Pseudo-Hermitian symmetric spaces and symmetric domains over nondegenerate cones, Hokkaido Math. J. 20 (1991), 213-239.

[L1] O. Loos, Bounded Symmetric Domains and Jordan Pairs, Univ. of California, Irvine, 1979.

[S1] W. Schmid, Die Randwerte holomorpher Funktionen auf hermitesch symmetrischen Räumen, Invent. Math. 9 (1969), 61-80.

[S2] - On the characters of discrete series (the hermitian symmetric case), ibid. 30 (1975), 47-144.

[U1] H. Upmeier, Jordan $\mathrm{C}^{*}$-Algebras and Symmetric Banach Manifolds, North-Holland, 1985.

[U2] - Jordan algebras and harmonic analysis on symmetric spaces, Amer. J. Math. 108 (1986), 1-25.

[U3] - Toeplitz operators on bounded symmetric domains, Trans. Amer. Math. Soc. 280 (1983), 221-237.

[U4] - Toeplitz $\mathrm{C}^{*}$-algebras on bounded symmetric domains, Ann. of Math. 119 (1984), 549-576.

[U5] -, Multivariable Toeplitz Operators and Index Theory, Birkhäuser, 1996.

[U6] A. \& J. Unterberger, Quantification et analyse pseudo-différentielle, Ann. Sci. École Norm. Sup. 21 (1988), 133-158.

[U7] H. Upmeier, Weyl quantization of symmetric spaces (I): Hyperbolic matrix domains, J. Funct. Anal. 96 (1991), 297-330.

[UU] A. Unterberger and H. Upmeier, The Berezin transform and invariant differential operators, Comm. Math. Phys. 164 (1994), 563-597. 